\title{
RESILIENNCIA ENTRE MULHERES IDOSAS E SUA ASSOCIAÇÃO COM O BEM-ESTAR ESPIRITUAL E O APOIO SOCIAL
}

\author{
Solange Beatriz Billig Garces ${ }^{1}$ \\ Michele Ferraz Figueiró ${ }^{2}$ \\ Dinara Hansen ${ }^{3}$ \\ Carolina Böettge Rosa ${ }^{4}$ \\ Angela Vieira Brunelli ${ }^{5}$ \\ Patrícia Dall'Agnol Bianchi6 \\ Patrícia Viana da Rosa ${ }^{7}$ \\ Bianca Bueno do Nascimento ${ }^{8}$
}

1 Licenciada em Educação Física. Doutora em Ciências Sociais. Professora Titular do PPG em Práticas Socioculturais e Desenvolvimento Social da Universidade de Cruz Alta - Unicruz, vinculada ao GIEEH - Grupo Interdisciplinar de Estudos do Envelhecimento Humano - CNPq/Unicruz. E-mail: sbgarces@hotmail.com.

2 Graduada em Física. Doutora em Física. Professora Adjunta ll do Centro de Ciências Humanas e Sociais da Unicruz, vinculada ao GIEEH. E-mail: mimiff25@yahoo.com.br.

3 Fisioterapeuta. Doutora em Gerontologia Biomédica. Professora do Centro de Ciências da Saúde da Unicruz, vinculada ao GIEEH. E-mail: dinarahansen@hotmail.com.

4 Nutricionista. Doutora em Gerontologia Biomédica, vinculada como pesquisadora do GIEEH. E-mail: carolboettge@gmail.com.

5 Nutricionista. Mestre em Extensão Rural. Professora do Centro de Ciências da Saúde da Unicruz, vinculada ao GIEEH. E-mail: angelavbrunelli@gmail.com.

6 Fisioterapeuta. Doutora em Ciências Biológicas.Professora Titular do PPG em Práticas Socioculturais e Desenvolvimento Social da Universidade de Cruz Alta - Unicruz, vinculada ao GIEEH. E-mail: patibianchi@yahoo.com.br.

7 Fisioterapeuta. Doutora em Gerontologia Biomédica.Professora Adjunta da Fundação Universidade Federal de Ciências da Saúde de Porto Alegre (UFCS/POA). E-mail: patriciarosa1@hotmail.com.

8 Acadêmica do Curso de Educação Física da Universidade de Cruz Alta - Unicruz. Estudante vinculada ao GIEEH - Grupo Interdisciplinar de Estudos do Envelhecimento Humano - CNPq/Unicruz. E-mail: biancabueno20@hotmail.com. 


\begin{abstract}
resumo
Este estudo descritivo observacional objetiva investigar o nível de resiliência entre mulheres idosas e sua associação com bem-estar espiritual e apoio social. A população constituiu-se de 241 mulheres. Os instrumentos aplicados foram: questionário para identificação de dados sociodemográficos; escala de resiliência; escala de apoio social e escala de bem-estar espiritual. A correlação entre o escore de cada escala e sua respectiva dimensão é indicada pelo coeficiente de correlação de Spearman. Para medir as associações entre resiliência, bem-estar espiritual e apoio social, foi utilizado o teste qui-quadrado. A análise estatística dos dados foi realizada no programa IBM SPSS 20.0. Os resultados indicaram que 50,2\% das idosas apresentam alto bem-estar espiritual, enquanto 75,9\% demonstraram alto apoio social e 79,7\% apresentaram alto nível de resiliência. Foi encontrada uma correlação positiva entre bem-estar espiritual e resiliência, e uma relação de dependência entre as escalas de resiliência e de apoio social. A associação entre resiliência e idade não foi significativa ( $p=0,137)$. Essas constatações demonstram que embora a vida tenha adversidade, o bem-estar espiritual e o apoio social fortalecem a resiliência.
\end{abstract}

palavras-chave

Resiliência psicológica. Apoio social. Idoso. Identidade de gênero.

Os últimos dados demográficos revelam que o país apresenta hoje um contingente de 20.590 .599 pessoas com mais de sessenta anos de idade, sendo que destes, 9.156 .112 são homens e 11.434.487 mulheres. Esses dados demonstram a grande prevalência de mulheres idosas no país, tornando os estudos nessa área extremamente relevantes (IBGE, 2010). Dentre os fatores que geraram uma modificação na pirâmide etária brasileira, com o aumento da população de mais de sessenta anos de idade e com um considerável número de mulheres idosas, constatados pelo último censo realizado no Brasil, destacam-se a diminuição da natalidade e da mortalidade infantil, a melhoria dos conhecimentos científicos em relação à saúde e condições sanitárias, como vacinas, medicamentos, higiene e cuidados preventivos (CAMARANO; KANSO; MELLO, 2004). 
Um dos fatores agravantes no envelhecimento é que esse é um processo naturalmente deletério, com involução física e mental. Portanto, nessa fase é comum o surgimento de doenças crônico-degenerativas que colaboram com a visão negativa do envelhecimento. É habitual então, o surgimento de incapacidades físicas e cognitivas. Para evitar isso é importante a manutenção de atividades físicas regulares e frequentes, especialmente as que mantenham a força e o tônus muscular (ALMEIDA; BEGER; WATANABE, 2007; CARVALHO FILHO; PAPALÉO NETTO; SALLES, 2006).

Entre as mulheres as doenças crônico-degenerativas são mais propensas, pois as mesmas têm maior tendência à obesidade e, portanto, doenças associadas como diabetes, dislipidemias, doenças cardíacas e hipertensão. Apesar disso, as mulheres vivem mais que os homens, pois se expõe menos aos fatores de risco do trabalho, da violência urbana, de acidentes de trânsito, do uso de tabaco e álcool e, em função de suas atitudes preventivas em relação às doenças, procuram bem mais os serviços médicos do que os homens (VERAS, 1996).

Uma das características da mulher idosa é a solidão, pois pelo fato de viver mais do que o homem há um maior número de mulheres idosas, especialmente acima dos oitenta anos (VERAS, 1996). Outro fator que corrobora para a solidão e tristeza no final da vida, além da viuvez e das doenças crônico-degenerativas, é a redução da renda. Dependendo da classe social e dos arranjos familiares, ser velha pode significar viver em grande pobreza, ou até na miséria, mesmo para aquelas originalmente de classe média, por tratar-se de uma geração de escassa participação no mercado de trabalho e, portanto, com poucos recursos pessoais de sobrevivência. Pode significar, também, falta de companheiro ou solidão mais frequente, devido ao maior número de viúvas, ao crescente número de separadas, ou de solteiras com filhos, mulheres chefiando famílias que nunca se constituíram completas (MOTTA, 1999).

Em pesquisa realizada sobre velhice de mulheres verificou-se que elas são mais propensas a experimentar a solidão, visto a viuvez ter se tornado uma realidade feminina típica da velhice (SANTOS, 2005). Esses aspectos são grandes contribuintes para a visão do envelhecimento como um momento difícil da vida, ou como um fardo para os parentes. Entretanto é preciso ter consciência de que mesmo sendo esse um período em que haja um declínio das capacidades físicas ou presença de doenças, o idoso pode continuar ativo a partir de suas possibilidades, mas a capacidade de ação deve partir do idoso.

O bem-estar tem a ver com as questões subjetivas, pois se refere à avaliação que a própria pessoa faz de sua vida, e o componente espiritual lhe dá o suporte para enfrentar as adversidades da vida que se tornam mais frequentes em épocas de maior fragilidade e solidão, que geralmente ocorrem no último 
ciclo da vida. Essa capacidade de ação para enfrentar as adversidades da vida chama-se resiliência e esse sentimento modifica o destino de muitos idosos. $\mathrm{E}$ as mulheres são realmente resilientes? Serão as idosas mais velhas mais resilientes que as idosas mais jovens? Hipotetiza-se que quanto maior o apoio espiritual e social mais resilientes se tornarão as idosas. Assim, as evidências dessa pesquisa, cujo objetivo é investigar o nível de resiliência entre mulheres idosas e sua associação com o bem-estar espiritual e apoio social, ajudarão a inibir ou ressaltar ações em relação a esses aspectos no envelhecimento visando tornar o referido período da vida mais aceitável e natural, como mais um ciclo da vida a ser plenamente vivenciado.

\section{Metodologia}

A pesquisa se refere a um estudo descritivo observacional com abordagem quantitativa. Para realização do projeto utilizou-se parte do banco de dados de um projeto de pesquisa maior intitulado "Identificação dos fatores associados à depressão entre idosos do município de Cruz Alta - RS", que recebeu aprovação pelo Comitê de Ética em Pesquisa (CEP) da Unicruz através do no 071/ 08.

A população do estudo constituiu-se dos sujeitos idosos do município de Cruz Alta -RS/Brasil, do sexo feminino, que totaliza 5.312 mulheres (IBGE, 2010) e a amostra constitui-se de 241 mulheres (4,55\% do total da população). Os critérios de inclusão foram mulheres com idade igual ou acima de 60 anos, selecionadas por conveniência, que não residissem em instituição de longa permanência e nem na zona rural. Os instrumentos de pesquisa utilizados para a constituição do banco de dados foram:

- Questionário para identificação de dados sociodemográficos da amostra: faixa etária, sexo, estado civil, escolaridade, aposentadoria e renda, condições de moradia e também de saúde, revelando autopercepção de saúde atualmente e ainda comparada a cinco anos atrás, além de relatar as doenças diagnosticadas mais prevalentes.

- Escala de Resiliência desenvolvida por Wagnild e Young (1993 apud PESCE et al., 2005), adaptada e validada por Pesce et al. (2005). A escala possui 25 itens descritos de forma positiva, com respostas tipo likert variando de 1 (discordo totalmente) a 7 (concordo totalmente). Os escores da escala oscilam de 25 a 175 pontos, com valores altos indicando elevada resiliência. E como ponto de corte considerou-se como menos resilientes aqueles com escores abaixo de 110.6, num total de 175 pontos. 
- Escala de Apoio Social, desenvolvida por Medical Outcomes Study (MOS)/Sociedade de Ciências Médicas (SHERBOURNE; STEWART, 1991). O questionário é composto por 19 itens com cinco dimensões funcionais de apoio social: material, afetivo, emocional, interação social positiva e informação. Para o cálculo dos escores, em cada uma das cinco dimensões de apoio social atribuiu-se pontos a cada opção de resposta. Para todas as perguntas, se apresentaram cinco opções de resposta: 1 (nunca); 2 (raramente); 3 (às vezes); 4 (quase sempre) e 5 (sempre). Para a Escala de Apoio Social, a análise e a interpretação dos dados foram baseadas em suas dimensões. Os escores obtidos pela soma dos pontos totalizados pelas perguntas de cada uma das dimensões foram divididos pelo escore máximo possível na mesma dimensão. O resultado da razão (total de pontos obtidos/pontuação máxima da dimensão) é multiplicado por 100 (GRIEP, 2005).

- Escala de Bem-Estar Espiritual (EBE - Spiritual Well-Being Scale), de Paulotzian e Ellison, adaptada por Marques (2003). É composta de 20 questões com pontuação de 1 a 6, dividida em duas subescalas, uma de bem-estar religioso (composta por 10 itens) e outra de bem-estar existencial (composta por 10 itens). Os pontos de corte sugeridos para o escore geral de bem-estar espiritual apresentam os intervalos de 20 a 40, 41 a 99 e 100 a 120, para baixo, moderado e alto bem-estar.

Inicialmente, realizou-se uma análise descritiva a partir do cálculo das frequências absoluta e percentual dos dados sociodemográficos, indicadores e autopercepção de saúde, e perfil de patologias das idosas investigadas. A média e o desvio padrão foram calculados para a soma total dos escores obtidos pelos itens de cada um dos instrumentos, assim como para suas respectivas dimensões.

A correlação item-total corrigida apresentou-se para cada item dos instrumentos de escala, representando a correlação entre um item e a soma do escore dos demais itens, e valores de correlação acima de 0,30 são considerados altos (DE VAUS, 2004).

O grau de correlação entre cada item e sua respectiva dimensão deu-se através do coeficiente de correlação de Spearman.

Para medir a associação entre a Escala de Bem-Estar Espiritual e a Escala de Resiliência, e entre a Escala de Apoio Social e a Escala de Resiliência, utilizou-se o Teste Qui-Quadrado de Pearson. Todas as análises estatísticas realizaram-se no programa IBM SPSS versão 20 e foi considerado o valor $p \leq 0,05$ para verificação da significância estatística. Também se calculou o coeficiente phi $(\phi)$, que é uma medida do grau de associação para dados categóricos. 


\subsection{Dados sociodemográficos e condições de saúde}

Os dados sociodemográficos das idosas investigadas aparecem na Tabela 1. A faixa etária predominante entre as mulheres pesquisadas foi dos 60 aos 79 anos (77,60\%) e 22,40\% com 80 anos ou mais. Em relação ao estado civil, predominaram idosas viúvas (44,8\%) em relação a casadas (37,8\%). Cabe destacar ainda, em relação aos dados sociodemográficos, que prevaleceram idosas com escolaridade de ensino fundamental incompleto $(68,5 \%), 12 \%$ analfabetas e $2,1 \%$ com ensino superior completo, demonstrando o quanto se negou às mulheres oportunidade de acesso à educação.

Tabela 1 - Dados sociodemográficos e econômicos.

\begin{tabular}{|c|c|c|c|}
\hline Categorias & Indicadores & $\mathrm{n}$ & $\%$ \\
\hline \multirow{3}{*}{ Faixa Etária } & 60 aos 69 anos & 99 & 41,1 \\
\hline & 70 aos 79 anos & 88 & 36,5 \\
\hline & 80 e mais & 54 & 22,4 \\
\hline \multirow{4}{*}{ Estado Civil } & Casada & 91 & 37,8 \\
\hline & Solteira & 27 & 11,2 \\
\hline & Viúva & 108 & 44,8 \\
\hline & Outra & 15 & 6,2 \\
\hline \multirow{8}{*}{ Escolaridade } & Analfabeta & 29 & 12,0 \\
\hline & Ens. Fund. Inc. & 165 & 68,5 \\
\hline & Ens. Fund. Comp. & 22 & 9,1 \\
\hline & Ens. Médio Inc. & 7 & 2,9 \\
\hline & Ens. Médio Completo & 12 & 5,0 \\
\hline & Ens. Sup. Inc. & 1 & 0,4 \\
\hline & Ens. Sup. Comp. & 5 & 2,1 \\
\hline & Pós-Graduação & 0 & 0,0 \\
\hline \multirow{2}{*}{ Aposentado } & Sim & 84 & 34,9 \\
\hline & Não & 157 & 65,1 \\
\hline
\end{tabular}

Continua 


\begin{tabular}{|c|c|c|c|}
\hline Categorias & Indicadores & $\mathrm{n}$ & $\%$ \\
\hline \multirow{6}{*}{ Renda } & Sem renda & 24 & 10,0 \\
\hline & 1 salário mínimo & 121 & 50,2 \\
\hline & De 2 a 4 salários mínimos & 84 & 34,9 \\
\hline & De 5 a 9 salários mínimos & 11 & 4,6 \\
\hline & De 10 a 15 salários mínimos & 1 & 0,4 \\
\hline & Mais de 15 salários mínimos & 0 & 0,00 \\
\hline
\end{tabular}

Fonte: Tabela elaborada pelas autoras.

Dentre as mulheres entrevistadas, 50,2\% vivem em família com um salário mínimo e 10,0\% não apresentam renda. Outro dado relevante é que $65,1 \%$ das mulheres idosas entrevistadas não são aposentadas. Esses dados revelam que essas mulheres idosas não tiveram acesso ao trabalho fora de casa, ou quando o tiveram não foi com carteira de trabalho assinada, o que lhes garantiria uma aposentadoria. Muitas dessas idosas que têm renda atualmente é resultado de pensões por viuvez.

As mulheres idosas entrevistadas relataram que $52,8 \%$ vivem com familiares, 30,2\% com o cônjuge ou companheiro e 17\% revelaram morar sozinhas. Em relação à situação da moradia, $86,3 \%$ são proprietárias, $5 \%$ alugam a residência, 3,3\% residem em casa de propriedade dos filhos, 2,9\% residem em casas cedidas e $2,5 \%$ em casa de propriedade de parentes.

Quando as mulheres se tornam muito idosas ou dependentes habitualmente precisam do apoio de parentes ou filhos e, geralmente acabam residindo na casa desses e abandonando o seu lar. A mudança de hábitos, a falta de seus pertences, a perda de suas histórias e a própria doença levam-nas a um grande vazio e tristeza, o que pode gerar depressão. Nesta direção, o apoio social e o bem-estar espiritual ajudarão a superar esse processo.

Em relação aos indicadores e autopercepção de saúde das idosas investigadas, 40,2\% percebem sua saúde como regular, 30,3\% como boa, 9,1\% como ótima, 8,3\% como muito boa, 7,1\% como ruim e 5\% como péssima. Quando questionadas sobre a sua percepção de saúde comparada há cinco anos, 29,5\% consideram boa, $27,4 \%$ regular, $15,4 \%$ ruim, $12,4 \%$ ótima, $11,6 \%$ muito boa e $3,7 \%$ péssima. Ao comparar os resultados vê-se que as idosas (53,5\%) consideram que sua saúde está melhor (conceito ótima, muito boa e boa) que há cinco anos $(47,7 \%)$, mesmo que (atualmente) estejam com mais idade do que anteriormente.

Dentre as patologias diagnosticadas por médicos, as idosas destacaram a hipertensão (70,1\%), a artrite e/ou doenças reumáticas (42,3\%), a osteoporose 
$(40,2 \%)$, a depressão $(36,1 \%)$, trombose/varizes $(32 \%)$, alergia $(32 \%)$, fraturas $(22,88 \%)$, úlcera/gastrite $(22,4 \%)$, constipação $(22,0 \%)$, diabetes $(20,3 \%)$, dislipidemia $(19,9 \%)$, doenças cardíacas $(34,4 \%)$, asma/bronquite $(14,1 \%)$, enfisema pulmonar (10\%), neoplasia $(5,4 \%)$, derrame $(4,1 \%)$ e doenças psiquiátricas $(2,1 \%)$. Destas idosas, $88,8 \%$ tomam medicamentos diariamente e somente $11,2 \%$ não, onde $56 \%$ tomam entre três ou mais medicamentos diferentes diariamente e $32,8 \%$ tomam até dois tipos de medicamentos.

Percebe-se que as entrevistadas são, na sua grande maioria, mulheres idosas com menos de 80 anos, pouco grau de escolaridade e que apresentam patologias, tais como hipertensão, doenças reumáticas, osteoporose e depressão. A depressão sendo uma doença estreitamente relacionada ao apoio social, pois a ajuda de familiares e amigos é de extrema importância para o não aparecimento da mesma. Cabe também ressaltar que grande parte das idosas compõe-se de mulheres independentes, que realizam suas atividades domésticas diárias, tais como cuidar e gerenciar sua casa.

\subsection{Bem-Estar Espiritual entre mulheres idosas}

Após aplicação da escala resultou que 49,8\% das mulheres idosas apresentaram baixo bem-estar espiritual. A média geral e o desvio padrão dos escores totais do instrumento foram de 98,15 e $\sigma=14,02$, respectivamente. Os valores da média e do desvio padrão da dimensão de Bem-Estar Existencial foram de 46,52 e $\sigma=7,52$, respectivamente; e a dimensão de Bem-Estar Religioso apresentou os valores de 51,63 e $\sigma=7,85$, respectivamente. O Quadro 1 mostra os valores obtidos dos parâmetros estatísticos que resumem e descrevem o comportamento entre o instrumento de Bem-Estar Espiritual e suas duas dimensões.

Quadro 1 - Correlação entre as dimensões do instrumento que avalia o nível de Bem-Estar Espiritual.

\begin{tabular}{|l|l|l|l|l|l|}
\hline \multicolumn{1}{|c|}{ Itens } & Média & $\begin{array}{c}\text { Desvio } \\
\text { padrão }\end{array}$ & $\begin{array}{c}\text { Correlação corrigida } \\
\text { item-valor total do } \\
\text { instrumento (Bem- } \\
\text {-Estar Espiritual) }\end{array}$ & $\begin{array}{c}\text { Bem-Estar } \\
\text { existencial* }\end{array}$ & $\begin{array}{c}\text { Bem-Estar } \\
\text { religioso* }\end{array}$ \\
\hline R1 & 4,76 & 1,70 & 0,38 & 0,23 & 0,45 \\
\hline R3 & 5,46 & 1,06 & 0,51 & 0,28 & 0,38 \\
\hline R5 & 4,92 & 1,43 & 0,53 & 0,34 & 0,53 \\
\hline
\end{tabular}


Continuação

\begin{tabular}{|c|c|c|c|c|c|}
\hline Itens & Média & $\begin{array}{l}\text { Desvio } \\
\text { padrão }\end{array}$ & $\begin{array}{l}\text { Correlação corrigida } \\
\text { item-valor total do } \\
\text { instrumento (Bem- } \\
\text {-Estar Espiritual) }\end{array}$ & $\begin{array}{l}\text { Bem-Estar } \\
\text { existencial }^{*}\end{array}$ & $\begin{array}{l}\text { Bem-Estar } \\
\text { religioso* }\end{array}$ \\
\hline $\mathrm{R} 7$ & 5,39 & 0,94 & 0,70 & 0,38 & 0,50 \\
\hline R9 & 4,95 & 1,38 & 0,58 & 0,29 & 0,52 \\
\hline $\mathrm{R} 11$ & 5,22 & 1,12 & 0,50 & 0,37 & 0,44 \\
\hline R13 & 4,94 & 1,36 & 0,48 & 0,25 & 0,51 \\
\hline R15 & 5,34 & 1,01 & 0,59 & 0,35 & 0,48 \\
\hline R17 & 5,29 & 1,04 & 0,56 & 0,38 & 0,53 \\
\hline R19 & 5,37 & 0,94 & 0,68 & 0,38 & 0,51 \\
\hline E2 & 4,08 & 1,12 & 0,26 & 0,15 & 0,20 \\
\hline E4 & 5,32 & 1,33 & 0,55 & 0,30 & 0,35 \\
\hline E6 & 3,67 & 1,36 & 0,24 & 0,24 & 0,17 \\
\hline E8 & 5,15 & 1,21 & 0,52 & 0,28 & 0,30 \\
\hline E10 & 5,07 & 1,01 & 0,56 & 0,40 & 0,31 \\
\hline E12 & 4,71 & 1,68 & 0,57 & 0,30 & 0,30 \\
\hline E14 & 4,97 & 1,04 & 0,53 & 0,33 & 0,31 \\
\hline E16 & 3,55 & 1,35 & 0,15 & 0,19 & 0,62 \\
\hline E18 & 4,73 & 0,94 & 0,57 & 0,40 & 0,33 \\
\hline E20 & 5,27 & 1,00 & 0,64 & 0,37 & 0,41 \\
\hline
\end{tabular}

Legenda das duas dimensões: R=Religioso e E=Existencial

${ }^{*}$ Correlação de Spearman significante ao nível de 0,01.

Fonte: Quadro elaborado pelas autoras.

A correlação item-total corrigida $\mathrm{r}=0,70$ mostra que existe uma correlação forte e positiva entre o escore do item R7 (Eu tenho uma relação pessoal significativa com Deus) e o escore combinado pelos outros demais 19 itens; e uma correlação fraca $(r=0,15)$ entre o item E16 (Eu sinto que a vida é cheia de conflitos e infelicidade) e o escore combinado pelos outros demais 19 itens. Isso significa dizer que as idosas valorizam mais a sua espiritualidade que as questões existenciais.

De acordo com a correlação de Spearman, os itens que apresentaram maior e menor correlação, respectivamente, com a dimensão BEE foram E10 
(Eu tenho um sentimento de bem-estar em relação à direção em que minha vida está encaminhada), r=0,40 e E2 (Eu não sei quem sou, de onde venho ou para onde vou), r=0,15; e com a dimensão BER foram E16 (Eu sinto que a vida é cheia de conflitos e infelicidade), r=0,62 e E6 (Eu me sinto intranquilo sobre o meu futuro), $r=0,17$. Essas constatações demonstram que embora a vida tenha suas adversidades, o bem-estar espiritual, através das dimensões religiosas e existenciais, as fortalece. A maior parte dos estudos permite concluir que o bem-estar espiritual é uma experiência de apoio, de fortalecimento buscado propositadamente pela pessoa para a realização de um enfrentamento de sucesso (MARQUES, 2003).

Mella et al. (2004) estudaram a relação de fatores associados ao bem-estar subjetivo de idosos e chegaram a conclusão que este é determinado pelo grau de apoio que os idosos recebem de sua rede de relações, influenciado pelo gênero e pela ausência de indícios depressivos. Embora a religiosidade e a espiritualidade sejam características permanentes no ser humano, elas são ainda bastante negligenciadas nos estudos gerontológicos. Entretanto isso é uma questão contraditória, pois existe a contribuição de alguns fatores empíricos para que se perceba que este é um fato presente entre as pessoas, não apenas idosas, tais como a grande maioria das pessoas que professa algum tipo de crença religiosa.

Várias pesquisas indicam que adultos e idosos tendem a valorizar suas crenças e dogmas religiosos nos momentos difíceis de suas vidas, quando não são controláveis, apelando para a sua fé (GOLDSTEIN; SOMMERHALDER, 2002). As referidas autoras citam estudos americanos que mostram metas das pessoas idosas mais ligadas a valores intrínsecos, ao autoconhecimento e a autoaceitação e sugere que à medida que a pessoa envelhece, ela começa a valorizar mais questões que satisfaçam suas necessidades psicológicas, assim como a religiosidade e a espiritualidade, tornando-se menos interessadas em questões materiais, as quais se tornam então superficiais para elas. As autoras concordam que crenças espirituais e religiosas parecem ser um dos poucos recursos que tendem a aumentar na velhice. Isso porque há um acréscimo de eventos não controláveis na velhice, instante em que o enfrentamento dessas questões através da espiritualidade e da religiosidade parece ser bastante efetivo. O bem-estar espiritual significa em que medida ocorre a abertura da pessoa para a dimensão espiritual permitindo a integração da espiritualidade com as outras dimensões da vida, maximizando seu potencial de crescimento e autorrealização (MARQUES, 2003).

As pessoas ao chegarem à meia-idade dão mais atenção aos aspectos internos do self, abrindo caminho aos sentimentos e comportamentos religiosos, 
assim, a religião para o idoso é um referencial pessoal. Entre as pessoas que têm práticas religiosas moderadas (não fanatismos), mas que participam ativamente nos trabalhos destas, apresentam objetivos de vida, possuem maior envolvimento com as pessoas, maior satisfação na vida e são menos estressadas e, talvez mais resilientes (CARVALHO; FERNANDEZ, 1996).

\subsection{Apoio social entre mulheres idosas}

Para Martins (2005) o conceito de apoio social está relacionado à interação dos indivíduos com o outro e sempre no sentido da promoção do bem-estar físico e psicológico. Assim, com a intenção de identificar o apoio social entre as idosas pesquisadas, aplicou-se o instrumento desenvolvido por Medical Outcomes Study (MOS)/Sociedade de Ciências Médicas (SHERBOURNE; STEWART, 1991).

A média e o desvio padrão dos escores totais do instrumento da Escala de Apoio Social foram 79,47 e $\sigma=17,76$, respectivamente. Uma vez que este instrumento possui cinco dimensões, os valores da média e do desvio padrão dos escores na dimensão Interação Social Positiva (ISP) foram, respectivamente, de 16,22 e $\sigma=4,21 ; 12,84$ e $\sigma=2,96$ na dimensão Afetiva (AF); 16,58 e $\sigma=4,30$ na dimensão Emocional (EM); 16,30 e $\sigma=4,35$ na dimensão Informativa (INF); e 17,53 e $\sigma=3,85$ na dimensão Material (MAT).

O Quadro 2 mostra os valores obtidos dos parâmetros estatísticos que resumem e descrevem o comportamento entre o instrumento de Apoio Social e suas cinco dimensões.

Quadro 2 - Correlação entre as dimensões do instrumento que avalia o nível de Apoio Social entre idosas.

\begin{tabular}{|l|l|l|l|l|l|l|l|l|}
\hline Itens & Média & $\begin{array}{c}\text { Desvio } \\
\text { padrão }\end{array}$ & $\begin{array}{c}\text { Correlação } \\
\text { item-valor } \\
\text { total do } \\
\text { instrumento } \\
\text { (Apoio Social) }\end{array}$ & ISP* & AF* $^{*}$ & $\mathbf{E M}^{*}$ & INF$^{*}$ & MAT $^{*}$ \\
\hline ISP1 & 4,18 & 1,07 & 0,81 & 0,85 & 0,74 & 0,73 & 0,70 & 0,70 \\
\hline ISP2 & 4,12 & 1,16 & 0,80 & 0,89 & 0,75 & 0,74 & 0,76 & 0,67 \\
\hline ISP3 & 4,05 & 1,16 & 0,79 & 0,90 & 0,77 & 0,75 & 0,73 & 0,65 \\
\hline ISP4 & 3,87 & 1,33 & 0,75 & 0,90 & 0,71 & 0,70 & 0,69 & 0,62 \\
\hline AF1 & 4,29 & 1,05 & 0,81 & 0,73 & 0,91 & 0,72 & 0,70 & 0,70 \\
\hline
\end{tabular}




\begin{tabular}{|l|l|l|l|l|l|l|l|l|}
\hline Itens & Média & $\begin{array}{c}\text { Desvio } \\
\text { padrão }\end{array}$ & $\begin{array}{c}\text { Correlação } \\
\text { item-valor } \\
\text { total do } \\
\text { instrumento } \\
\text { (Apoio Social) }\end{array}$ & ISP* & AF* & EM* & INF* & MAT* $^{*}$ \\
\hline AF2 & 4,25 & 1,05 & 0,80 & 0,75 & 0,94 & 0,74 & 0,73 & 0,68 \\
\hline AF3 & 4,30 & 1,03 & 0,81 & 0,75 & 0,93 & 0,77 & 0,71 & 0,66 \\
\hline E1 & 4,04 & 1,24 & 0,82 & 0,76 & 0,74 & 0,92 & 0,75 & 0,67 \\
\hline E2 & 4,10 & 1,20 & 0,86 & 0,73 & 0,74 & 0,94 & 0,83 & 0,70 \\
\hline E3 & 4,25 & 1,06 & 0,87 & 0,73 & 0,75 & 0,90 & 0,82 & 0,73 \\
\hline E4 & 4,20 & 1,12 & 0,86 & 0,72 & 0,75 & 0,89 & 0,81 & 0,70 \\
\hline IN1 & 4,14 & 1,16 & 0,85 & 0,74 & 0,74 & 0,81 & 0,90 & 0,68 \\
\hline IN2 & 4,13 & 1,15 & 0,86 & 0,74 & 0,77 & 0,83 & 0,91 & 0,71 \\
\hline IN3 & 3,90 & 1,35 & 0,70 & 0,68 & 0,65 & 0,72 & 0,92 & 0,57 \\
\hline IN4 & 4,14 & 1,19 & 0,84 & 0,73 & 0,73 & 0,81 & 0,87 & 0,76 \\
\hline MAT1 & 4,43 & 1,05 & 0,77 & 0,66 & 0,68 & 0,70 & 0,66 & 0,85 \\
\hline MAT2 & 4,52 & 0,94 & 0,74 & 0,64 & 0,67 & 0,67 & 0,65 & 0,81 \\
\hline MAT3 & 4,17 & 1,28 & 0,73 & 0,66 & 0,66 & 0,68 & 0,67 & 0,94 \\
\hline MAT4 & 4,41 & 1,05 & 0,69 & 0,60 & 0,60 & 0,60 & 0,59 & 0,85 \\
\hline
\end{tabular}

Legenda das cinco dimensões: ISP=Interação Social Positiva, AF=Afetiva, E=Emocional , IN=Informativa e MAT=Material. *Correlação de Spearman significante ao nível de 0,01.

Fonte: Quadro elaborado pelas autoras.

Neste estudo, a correlação item-total corrigida $\mathrm{r}=0,87$ mostra que existe uma correlação forte e positiva entre o escore do item E3 (Contar com alguém que compreenda seus problemas) e o escore combinado pelos outros demais 18 itens. A variação da correlação item-total corrigida entre os 19 itens foi de 0,69 a 0,87 .

Segundo a correlação de Spearman entre cada um dos 19 itens e as cinco dimensões do instrumento, os itens que apresentaram maior e menor correlação com a dimensão ISP foram ISP3 (Contar com alguém com quem relaxar), $\mathrm{r}=0,90$ e MAT4 (Contar com alguém para preparar suas refeições se você não puder prepará-las), $\mathrm{r}=0,60$, respectivamente; com a dimensão $\mathrm{AF}$ foram AF2 (Contar com alguém que lhe dê um abraço), r=0,94 e MAT4 (Contar com alguém para preparar suas refeições se você não puder prepará-las), r=0,60, 
respectivamente; com a dimensão EM foram E2 (Contar com alguém que compreenda seus problemas), $\mathrm{r}=0,94$ e MAT4 (Contar com alguém para preparar suas refeições se você não puder prepará-las), $\mathrm{r}=0,60$, respectivamente; com a dimensão INF foram IN3 (Alguém de quem você realmente quer conselhos), $\mathrm{r}=0,92$ e MAT4 (Contar com alguém para preparar suas refeições se você não puder prepará-las), $r=0,59$, respectivamente; e com a dimensão MAT foram MAT3 (Alguém para ajudá-lo nas tarefas diárias), r=0,94 e IN3 (Alguém de quem você realmente quer conselhos), $\mathrm{r}=0,57$, respectivamente.

Estes resultados sugerem que as mulheres idosas entrevistadas buscam a companhia de alguém para conversar e contar seus problemas, receber conselhos, afeto e carinho. Isso se torna algo mais importante do que ter a companhia apenas para o preparo de uma refeição, por exemplo. Obtiveram-se estes resultados em razão de que a maioria das idosas entrevistadas são independentes, autônomas e capazes de realizarem suas próprias atividades cotidianas. Essa ideia se reforça nas seguintes colocações: ao verificar que o apoio social se refere a funções desempenhadas por grupos ou pessoas significativas (familiares, amigos ou vizinhos) para o indivíduo em determinadas situações da vida (MARTINS, 2005). Esse apoio é percebido a partir da subjetividade de cada um e se traduz pela crença generalizada que os indivíduos desenvolveram de que os outros se interessam por eles, que estão disponíveis quando eles precisam, e isso suscita satisfação quanto às relações que têm (MARTINS, 2005).

A classe social está relacionada com o suporte social na velhice. No entanto, estas diferenças não se manifestam entre todos os tipos ou dimensões do suporte social. Ao contrário, os idosos com altas rendas e altos níveis de educação diferem daqueles nos mais baixos escalões somente em termos de contatos com amigos (e não com familiares), da frequência do suporte fornecido para outros, e na satisfação com suporte (RAMOS, 2002). Também esse autor observa que as pessoas casadas ou com companheiros vivos possuem maior apoio social e suporte na hora em que mais necessitam, como nos momentos de doenças e incapacidades, do que os(as) idosos(as) viúvos(as) (RAMOS, 2002).

Portanto, pode-se dizer que o apoio social entre as idosas está ligado ao grupo de relações, primeiramente o familiar e íntimo e secundariamente as relações externas de amizades e redes sociais, já que rede social (social network) pode ser definida como o grupo de pessoas com as quais o indivíduo mantém contato ou alguma forma de vínculo social, que podem ou não oferecer ajuda em diversas situações ao longo da vida (GRIEP, 2005). 


\subsection{Resiliência entre mulheres idosas}

Como mencionado anteriormente, o envelhecimento é um ciclo da vida que pode ser ativo e aproveitado como mais uma fase da vida. Entretanto, ele se caracteriza por ser uma fase bastante heterogênea. Para alguns pode ser um momento de continuidade, de ativa participação social, mas para outros pode ser um momento de enfrentamento de doenças, de fortes declínios da capacidade física e cognitiva, de solidão e depressão. O envelhecimento, mesmo sendo um processo individual e diferenciado traz algumas características que são comuns a todos, como o declínio das funções sensoriais, motoras, as mudanças nas relações, a perda de parentes, familiares e amigos, e essa constatação deixa claro a finitude da vida, o que geralmente provoca sensação de solidão, de tristeza e a aceitação dessa condição, para manutenção do equilíbrio psicológico dos indivíduos passa por um processo chamado de resiliência. Portanto, para enfrentar as adversidades da vida e as situações que não podem ser modificadas, lança-se mão de um comportamento chamado resiliência. A resiliência é uma capacidade que:

[...] implica adaptação em face do perigo, a capacidade de sair vencedor de uma prova que poderia ter sido traumática, com uma força renovada. Uma polarização exclusiva nos aspectos vulneráveis dos indivíduos e nos fatores de risco do seu contexto de vida pode levar a que se negligencie os contributos do estudo dos sujeitos que demonstram procedimentos adaptativos e de recursos psíquicos que os preservam dos aspectos psicotóxicos do seu ambiente. (LARANJEIRAS, 2007, p. 327).

Referenda ainda este autor que o núcleo central da resiliência é o fato de trabalhar os potenciais e os modos dos sujeitos em face do perigo.

O conceito de resiliência tem sido utilizado em diferentes ações sociais, educativas e de saúde e englobam indivíduos de todas as idades, da infância à velhice. A resiliência está ligada ao desenvolvimento e ao crescimento humano. As condutas de resiliência requerem fatores de resiliência e ações. As condutas resilientes supõem a presença e a interação dinâmica de fatores e esses vão mudando nas diferentes etapas do desenvolvimento. As situações de adversidade não são estáticas, mudam e requerem mudanças nas condutas resilientes. Assim, a conduta resiliente exige se preparar, viver e aprender com as experiências adversas, como mudança de país, doença ou abandono (MELILLO; OJEDA, 2006). Portanto, conforme esses autores, pode-se definir resiliência como sendo "a capacidade humana para enfrentar vencer e ser fortalecido ou transformado por experiências de adversidade" e a maioria 
das demais definições do conceito de resiliência é variação desta. Esses autores reforçam que o papel da resiliência é desenvolver a capacidade humana de enfrentar, vencer e sair fortalecido de situações adversas e transformado. Constitui em um processo que excede o simples superar estas experiências, uma vez que permite sair fortalecido por elas, o que necessariamente afeta a saúde mental (MELILLO; OJEDA, 2006). Nesse sentido, Fortes, Portuguez e Argimon (2009) realizaram pesquisa com 86 idosos de ambos os sexos, com idade entre 60 e 90 anos, para estudar a resiliência do idoso e sua relação com as variáveis sociodemográficas e funções cognitivas, concluindo que quanto mais altos os escores de resiliência, maiores os escores no desempenho cognitivo entre os idosos.

Poletto e Kohler (2008) estudaram contextos ecológicos, de acordo com a Teoria de Urie Bronfenbrenner, que são promotores de resiliência, fatores de risco e de proteção e chegaram à conclusão que qualquer que seja o contexto (família ou instituição) pode configurar como fator de risco ou proteção, pois dependerá da qualidade das relações, do afeto e da reciprocidade de conexões positivas para se acionarem processos de resiliência que favoreçam a melhoria da qualidade de vida, da saúde e da adaptação das pessoas as suas condições de vida em sociedade. Silva et al. (2012, p. 111) analisaram as "características resilientes por meio do perfil emocional de idosos de um grupo de atendimento multidisciplinar" e concluíram que "quando aprendem a lidar com as limitações e obstáculos decorrentes do envelhecimento, os idosos vivenciam uma velhice saudável."

Em estudo realizado sobre a resiliência em cuidadores de idosos com Alzheimer (onde a maioria eram mulheres), observou-se que embora alguns dos cuidadores do estudo apresentam-se fatigados e sobrecarregados, o que lhes motiva a continuar e persistir na tarefa do cuidado com certeza é a sua capacidade de resistir aos desafios e provações que a vida lhes oferece, pois a maioria deles $(83,3 \%$ ) apresentaram uma alta disposição à resiliência (GARCES et al., 2012).

O conceito de resiliência distingue três componentes fundamentais que são a adversidade (a noção de adversidade, trauma, risco ou ameaça ao desenvolvimento humano); a adaptação positiva (ou superação da adversidade) e o processo (que considera a dinâmica entre mecanismos emocionais, cognitivos e socioculturais que influem no desenvolvimento humano.) (MELILLO; OJEDA, 2006). Portanto, para investigar o nível de resiliência das idosas dessa pesquisa aplicou-se o instrumento de Escala de Resiliência. Na análise descritiva, obteve-se que 79,7\% das idosas entrevistadas apresentaram um alto nível de 
resiliência enquanto que 20,3\% uma baixa resiliência. Isso significa dizer que frente às adversidades, a maioria das idosas consegue buscar força e superação. A correlação item-total corrigida $r=0,74$ mostra que existe uma correlação forte e positiva entre o escore do item ER19 (Eu posso geralmente olhar uma situação de diversas maneiras) e o escore combinado pelos outros demais 24 itens e uma correlação muito fraca $(\mathrm{r}=0,08)$ entre o item ER1 (Quando eu faço planos eu levo até o fim) e o escore combinado pelos demais 24 itens, conforme demonstrado no Quadro 3.

Quadro 3 - Consistência interna do instrumento Escala de Resiliência.

\begin{tabular}{|l|l|l|l|}
\hline \multicolumn{1}{|c|}{ Itens } & \multicolumn{1}{|c|}{ Média } & Desvio padrão & \multicolumn{1}{|c|}{$\begin{array}{c}\text { Correlação corrigida item-valor } \\
\text { total do instrumento (Resiliência) }\end{array}$} \\
\hline ER1 & 4,42 & 1,04 & 0,08 \\
\hline ER2 & 5,80 & 1,62 & 0,59 \\
\hline ER3 & 5,75 & 1,54 & 0,69 \\
\hline ER4 & 5,66 & 1,83 & 0,67 \\
\hline ER5 & 5,94 & 1,55 & 0,66 \\
\hline ER6 & 5,74 & 1,80 & 0,61 \\
\hline ER7 & 6,21 & 1,32 & 0,51 \\
\hline ER8 & 5,32 & 1,82 & 0,45 \\
\hline ER9 & 6,31 & 1,26 & 0,69 \\
\hline ER10 & 5,27 & 1,95 & 0,62 \\
\hline ER11 & 6,02 & 1,53 & 0,62 \\
\hline ER12 & 4,50 & 2,21 & 0,12 \\
\hline ER13 & 5,50 & 1,90 & 0,46 \\
\hline ER14 & 6,02 & 1,55 & 0,67 \\
\hline ER15 & 6,12 & 1,39 & 0,67 \\
\hline ER16 & 6,08 & 1,50 & 0,70 \\
\hline ER17 & 5,67 & 0,55 \\
\hline ER18 & 5,93 & 0,64 \\
\hline ER19 & 6,14 & 1,45 & 0,74 \\
\hline
\end{tabular}

Continua 
Continuação

\begin{tabular}{|l|l|l|l|}
\hline \multicolumn{1}{|c|}{ Itens } & \multicolumn{1}{|c|}{ Média } & Desvio padrão & $\begin{array}{c}\text { Correlação corrigida item-valor } \\
\text { total do instrumento (Resiliência) }\end{array}$ \\
\hline ER20 & 5,69 & 1,60 & 0,72 \\
\hline ER21 & 4,83 & 2,13 & 0,26 \\
\hline ER22 & 6,09 & 1,39 & 0,72 \\
\hline ER23 & 5,34 & 1,94 & 0,36 \\
\hline ER24 & 5,81 & 1,52 & 0,73 \\
\hline ER25 & 5,51 & 1,87 & 0,61 \\
\hline
\end{tabular}

Legenda do instrumento: ER=Escala de Resiliência.

${ }^{*}$ Correlação de Spearman significante ao nível de 0,01.

Fonte: Quadro elaborado pelas autoras.

Outro estudo também demonstra que ao evidenciar na vivência dos idosos a espiritualidade como forte sinalizador da resiliência, solicita-se, de fato, uma proposta de espiritualidade, ou seja, uma série de valores e atitudes humanas e cristãs importantes para se viver, especialmente a última etapa, com maior bem-estar (SILVA, 2007).

\subsection{Associação entre Resiliência, Bem-Estar Espiritual e Apoio Social}

A Tabela 2 resume os três instrumentos de pesquisa utilizados de acordo com os dados de frequências absoluta e percentual obtidos.

Tabela 2 - Dados resumidos dos três instrumentos de pesquisa.

\begin{tabular}{|l|l|l|l|}
\hline \multicolumn{1}{|c|}{ Instrumentos } & \multicolumn{1}{|c|}{ Indicadores } & \multicolumn{1}{c|}{ n } & \multicolumn{1}{c|}{$\%$} \\
\hline \multirow{2}{*}{ Apoio Social } & Baixo & 58 & 24,1 \\
\cline { 2 - 4 } & Alto & 183 & 75,9 \\
\hline \multirow{2}{*}{ Bem-Estar Espiritual } & Baixo & 120 & 49,8 \\
\cline { 2 - 5 } & Alto & 121 & 50,2 \\
\hline \multirow{2}{*}{ Resiliência } & Baixo & 49 & 20,3 \\
\cline { 2 - 5 } & Alto & 192 & 79,7 \\
\hline Total & & 241 & 100 \\
\hline
\end{tabular}

Fonte: Tabela elaborada pelas autoras. 
De acordo com a Tabela 3, observa-se que 120 idosas (49,8\% do total) encontram-se no indicador baixo do instrumento de Escala de Bem-Estar Espiritual e, destes, 33 (13,7\% do total) pertencem ao indicador baixo e 87 (36,1\% do total) pertencem ao indicador alto do instrumento de Escala de Resiliência. Enquanto que 121 idosas (50,2\% do total) estão no indicador alto do instrumento de Escala de Bem-Estar Espiritual e, destes, 16 (6,6\% do total) pertencem ao indicador baixo e 105 (43,6\% do total) pertencem ao indicador alto do instrumento de Escala de Resiliência.

Tabela 3 - Tabela de contingência das Escalas de Resiliência e de Bem-Estar Espiritual.

\begin{tabular}{l|l|l|l|l}
\hline \multirow{2}{*}{$\begin{array}{l}\text { Escala de } \\
\begin{array}{l}\text { Bem-Estar } \\
\text { Espiritual }\end{array}\end{array}$} & \multicolumn{3}{|c|}{ Escala de Resiliência } & \multirow{2}{*}{ Total } \\
\cline { 2 - 5 } & Indicadores & \multicolumn{1}{|c|}{ Baixo } & \multicolumn{1}{c}{ Alto } & \\
\cline { 2 - 5 } & Baixo do total & 33 & 87 & 120 \\
\cline { 2 - 5 } & Alto & 16,7 & 36,1 & 49,8 \\
\cline { 2 - 5 } & \% do total & 6,6 & 105 & 50,2 \\
\hline Total & 49 & 192 & 241 \\
\hline$\%$ & 20,3 & 79,7 & 100 \\
\hline
\end{tabular}

Fonte: Tabela elaborada pelas autoras

Utilizou-se o teste estatístico qui-quadrado de Pearson para medir a associação entre as variáveis categóricas dos instrumentos de Escala de Resiliência e de Bem-Estar Espiritual. $\mathrm{O}$ valor da estatística qui-quadrada foi de 7,58. O valor $\mathrm{p}=0,005$ encontrado é altamente significativo para o nível de significância $\alpha=0,05$, indicando que existe uma relação de dependência entre as Escalas de Resiliência e de Bem-Estar Espiritual. O coeficiente $f i(\phi)$ encontrado foi de 0,18 indicando a presença de correlação fraca entre as Escalas BEE e Resiliência.

De acordo com a Tabela 4, observa-se que 58 idosas (24,1\% do total) encontram-se no indicador baixo do instrumento de Escala de Apoio Social e, destes, 28 (11,6\% do total) pertencem ao indicador baixo e 30 (12,4\% do total) pertencem ao indicador alto do instrumento de Escala de Resiliência. Enquanto que 183 idosas (75,9 \% do total) estão no indicador alto do instrumento de Escala de Apoio Social e, destes, 21 (8,7\% do total) pertencem ao indicador baixo e 162 (67,2\% do total) pertencem ao indicador alto do instrumento de Escala de Resiliência. 
Tabela 4 - Tabela de Contingência das Escalas de Resiliência e de Apoio Social.

\begin{tabular}{|c|c|c|c|c|}
\hline \multirow{6}{*}{$\begin{array}{l}\text { Escala de Apoio } \\
\text { Social }\end{array}$} & \multicolumn{3}{|c|}{ Escala de Resiliência } & \multirow{2}{*}{ Total } \\
\hline & Indicadores & Baixo & Alto & \\
\hline & Baixo & 28 & 30 & 58 \\
\hline & $\%$ do total & 11,6 & 12,4 & 24,1 \\
\hline & Alto & 21 & 162 & 183 \\
\hline & $\%$ do total & 8,7 & 67,2 & 75,9 \\
\hline Total & & 49 & 192 & 241 \\
\hline$\%$ & & 20,3 & 79,7 & 100 \\
\hline
\end{tabular}

Fonte: Tabela elaborada pelas autoras.

O valor da estatística qui-quadrada encontrado para medir a associação entre as variáveis categóricas dos instrumentos de Escala de Resiliência e de Apoio Social foi de 36,82 . O valor de $p=0,000$ é altamente significativo para o nível de significância $\alpha=0,05$, indicando que existe também uma relação de dependência entre as Escalas de Resiliência e de Apoio Social. O coeficiente phi $(\phi)$ foi de 0,39 indicando a presença de correlação moderada entre as Escalas Apoio Social e Resiliência. A associação entre a faixa etária das idosas e a Escala de Resiliência não apresentou nenhuma significância estatística ( $\mathrm{p}=0$, 137) revelando que não há nenhuma dependência entre idade e resiliência.

\section{Conclusão}

A pesquisa revelou uma prevalência de mulheres idosas, com menos de 80 anos, pouco estudo, baixa renda, apresentando patologias como hipertensão, doenças reumáticas, osteoporose e a mais preocupante, a depressão. Essa última doença está estreitamente relacionada com o apoio social, pois a família e amigos podem colaborar para que essa não apareça. Cabe também ressaltar que em sua maioria, as idosas são independentes e fazem suas atividades de vida diária, cuidando e gerenciando a sua casa.

As evidências dessa pesquisa revelaram um alto bem-estar espiritual e, portanto, essas constatações demonstram, que embora a vida tenha adversidades, o bem-estar espiritual, através das dimensões religiosas e existenciais as fortalece. Também demonstraram ter um alto apoio social. As idosas querem contar com a companhia de alguém, para conversarem e contarem seus problemas, receberem conselhos, afeto e carinho. Portanto, percebe-se que o 
apoio social entre as idosas se liga ao grupo de relações, primeiramente ao familiar e íntimo e secundariamente às relações externas de amizades e redes sociais. Da mesma forma apresentaram alto nível de resiliência. Embora os testes estatísticos revelem associações fracas e moderadas, pode-se afirmar que bem-estar espiritual, apoio social e resiliência constituem aspectos dependentes um dos outros.

Essas conclusões do estudo deixam como principal aspecto a importância das relações afetivas de familiares e grupo social entre idosos, o que torna importante a contribuição que as Universidades, através dos programas Universidades Abertas a Terceira Idade proporcionam aos idosos e, da mesma forma, as atividades de grupos de terceira idade que as demais instituições como SESC, igrejas e prefeituras oferecem aos idosos. Esses espaços permitem que os idosos, através do apoio social tornem-se resilientes às agruras que a vida carrega consigo e, de forma mais intensa, no período do envelhecimento.

Com o aumento expressivo do número de idosos há necessidade de estudos sobre temáticas como espiritualidade, bem-estar e apoio social na velhice, já que esses são fatores determinantes para a presença da resiliência entre idosos.

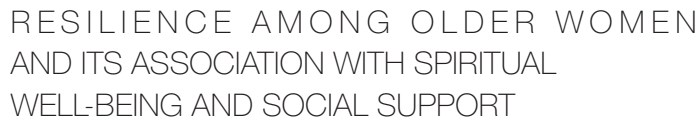

abstract

This observational descriptive study aims at investigating the level of resilience among older women and its association with spiritual well-being and social support. The population consisted of 241 women. The instruments applied were: questionnaire to identification of sociodemographic data; resilience scale; social support scale and spiritual well-being scale. The correlation between the score of each scale and its respective dimension is indicated by the Spearman correlation coefficient. To measure the associations between resilience, spiritual well-being and social support, chi-square test was used. The statistical analysis of the data was done in the IBM SPSS 20.0. The results indicated that $50.2 \%$ of older women present high spiritual well-being, while $75.9 \%$ demonstrated high social support and $79.7 \%$ presented high level of resilience. It was found a positive association between spiritual well-being and resilience, and a dependence relation between the resilience and social support scales. The association between resilience and age wasn't significant ( $p=0.137$ ). 
These findings demonstrate that although life has its adversities, the spiritual well-being and social support strengthen the resilience.

keywords

Psychological resilience. Social support. Older women. Gender identity.

referências

ALMEIDA, Maria Helena Morgani de; BEGER, Maria Lucia Martuscelli; WATANABE, Helena Akemi Wada. Memory training for the elderly: a health promotion strategy. Interface: comunicação, saúde e educação, São Paulo, v. 11, n. 22, p. 271-280, maio/ago. 2007.

CAMARANO, Ana Amélia; KANSO, Solange; MELLO, Juliana Leitão e. Como vive o idoso brasileiro? In: CAMARANO, Ana Amélia (Org.). Os novos idosos brasileiros muito além dos 60? Rio de Janeiro: IPEA, 2004. p. 25-73.

CARVALHO FILHO, Eurico Thomas; PAPALÉO NETTO, Matheus; SALLES, Renata Freitas Nogueira. Fisiologia do envelhecimento: geriatria, fundamentos, clínica e terapêutica. 2. ed. São Paulo: Atheneu, 2006.

CARVALHO, Valdecir de Fátima Cardozo; FERNANDEZ, Maria Elida Davila. Depressão no idoso. In: PAPALÉO NETTO, M. Gerontologia: a velhice e o envelhecimento em visão globalizada. São Paulo: Atheneu, 1996. p. 160-173.

DE VAUS, David. Survey in Social Research. London: Routledge, 2004.

FORTES, Tatiane Favarin Rech; PORTUGUEZ, Mirna Wetters; ARGIMON, Irani Iracema de Lima. A resiliência em idosos e sua relação com variáveis sociodemográficas e funções cognitivas. Estudos de Psicologia, Campinas, n. 26, v. 4, p. 455-463, out./dez. 2009.

GOLDSTEIN, Lucila de Lourdes Lucchino; SOMMERHALDER, Cinara. Religiosidade, Espiritualidade e Significado Existencial na Vida Adulta e Velhice. In: FREITAS, E. V. et al. (Org.). Tratado de Geriatria e Gerontologia. Rio de Janeiro: Guanabara Koogan, 2002. p. 950-956.

GARCES, Solange Beatriz Billig et al. Avaliação da Resiliência de Cuidador de Idosos com Alzheimer. Revista Brasileira de Geriatria e Gerontologia, Rio de Janeiro, v. 15, n. 2, p. 335-352, abr./jun. 2012.

GRIEP, Rosane Harter. Validade de constructo de escala de apoio social do Medical Outcomes Study adaptada para o português no Estudo Pró-Saúde. Cadernos de Saúde Pública, Rio de Janeiro, v. 21, n. 3, p. 703-714, maio/jun. 2005.

INSTITUTO BRASILEIRO DE GEOGRAFIA E ESTATíSTICA (IBGE). Sintese de Indicadores Sociais: uma análise das condições de vida da população brasileira. Rio de Janeiro: IBGE, 2010.

LARANJEIRAS, Carlos António Sampaio de Jesus. Do vulnerável ser ao resiliente envelhecer: revisão de literatura. Psicologia: teoria e pesquisa, Brasília, DF, v. 23, n. 3, p. 327-332, jul./set. 2007.

MARQUES, Luciana Fernandes. A saúde e o bem-estar espiritual em adultos porto-alegrenses. Psicologia, Ciência e Profissão, Brasília, DF, v. 23, n .2, p. 56-65, jun. 2003.

MARTINS, Rosa Maria. A relevância do apoio social na velhice. Revista Millenium, Viseu, v. 10, n. 31, p. 128-34, maio 2005. 
MELLA, R. et al. Factores asociados al bienestar subjetivo em El adulto mayor. Psykhe, Santiago, v. 13, n. 1, p. 79-89, maio 2004.

MELILLO, Aldo; OJEDA, Elbio Néstor Suárez (Org.). Resiliência: descobrindo as próprias fortalezas. Tradução de Valério Campos. Porto Alegre: Artmed, 2006.

MOTTA, Alda Brito. As dimensões de gênero e classe social na análise envelhecimento. Cadernos Pagu, São Paulo, n. 13, p. 191-221, 1999.

PESCE, Renata P. et al. Adaptação transcultural, confiabilidade e validade da escala de resiliência. Cadernos de Saúde Pública, Rio de Janeiro, v. 21, n. 2, p. 436-448, mar./abr. 2005.

POLETTO, Michele; KOLLER, Silvia Helena. Contextos ecológicos: promotores de resiliência, fatores de risco e de proteção. Estudos de Psicologia, Campinas, n. 25, v. 3, p. 405-416, jul./set. 2008.

RAMOS, Marília P. Apoio social e saúde entre idosos. Sociologias, Porto Alegre, v. 4, n. 7, p. 156-175, jan./jun. 2002.

SANTOS, Roberta Mercadante. Saidas, invenções e poderes: estudos sobre velhices de mulheres. 2005. 108 f. Dissertação (Mestrado em Política Social)-Universidade Federal Fluminense, Rio de Janeiro, 2005.

SHERBOURNE C.D.; STEWART A.L. The MOS social support survey. Social Science \& Medicine, Oxford, v. 32, n. 6, p. 705-714, 1991.

SILVA, Antônio Itamar. Envelhecimento: resiliência e espiritualidade. Diálogos Possíveis, Bahia, v.1, p. 191-209, jan./jun. 2007.

SILVA, Emília Amélia Pinto Costa da et al.Resiliência e saúde: uma análise da qualidade de vida em idosos. ConScientiae Saúde, São Paulo, v. 11, n. 1, p. 111-118, 2012

VERAS, Renato. Atenção preventiva ao idoso: uma abordagem de Saúde Coletiva. In: PAPALÉO NETTO, Matheus. Gerontologia: a velhice e o envelhecimento em visão globalizada. São Paulo: Atheneu, 1996. p. 383-393. 